\title{
Reator compartimentado anaeróbio/aeróbio: Sistema de baixo custo para tratamento de esgotos de pequenas comunidades
}

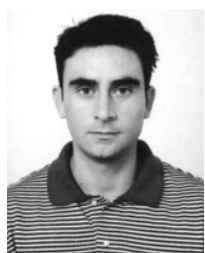

\author{
Gustavo H. R. da Silva' \& Edson A. A. Nour ${ }^{2}$ \\ ${ }^{1}$ Doutorando em Hidráulica e Saneamento. EESC/USP. Rua Sérgio Fernando Paranhos Fleury 49, Bairro Jd. Santa \\ Helena - CEP: 13566-430, São Carlos, SP. E-mail: gustavoh@sc.usp.br (Foto) \\ 2 Dept. de Saneamento e Ambiente, Faculdade de Engenharia Civil - UNICAMP, Rua Dr. Olímpio da Silva Miranda 126 , \\ Barão Geraldo, CEP 13083-010, Campinas, SP, Email:ednour@fec.unicamp.br
}

Protocolo 106 - 25/6/2003 - Aprovado em 17/8/2004

\begin{abstract}
Resumo: No Brasil são conhecidas e usadas várias técnicas de tratamento de esgoto, desde os mais sofisticados sistemas de tratamento aos mais simples processos. Uma alternativa economicamente viável aos sistemas de tratamento de esgotos sanitários é o processo anaeróbio, o qual tem recebido especial atenção nas pesquisas desenvolvidas no País, notadamente os reatores anaeróbios de alta taxa, unidades compactas e com boa eficiência de tratamento. Dentre as alternativas tecnológicas capazes de proporcionar as referidas variações das condições ambientais necessárias à ocorrência dos processos, está o uso de reatores compartimentados anaeróbio/aeróbio. Este trabalho apresenta, como alternativa, um reator compartimentado anaeróbio/ aeróbio, tratando esgoto sanitário, como possibilidade de ser utilizado em pequenas comunidades urbanas e rurais. $O$ desempenho do sistema foi avaliado em diversos tempos de detenção hidráulica (TDH), nos quais os melhores valores de Demanda Química de Oxigênio Total (DQO total $\left._{1}\right)-73,7 \%$ e Sólidos em Suspensão Totais (SST) - 78,8\%, foram obtidos com $\mathrm{TDH}_{\text {total }}$ de 8 horas (4 horas para a fase anaeróbia e 4 horas para a fase aeróbia). O reator compartimentado apresentou a característica de estabilidade operacional necessária aos sistemas de baixo custo.
\end{abstract}

Palavras-chave: reator compartimentado, processo anaeróbio/aeróbio, sistema de baixo custo, comunidades rurais

\section{Anaerobic/aerobic baffled reactor: Low cost system for sewage treatment in small communities}

\begin{abstract}
Several techniques of sewage treatment, from the most sophisticated to the simplest processes, are known in Brazil. An economically viable alternative for the systems of treatment of sanitary sewage is the anaerobic process, which has been given special attention in Brazilian research programs, mainly in the field of high rate anaerobic reactors as they are compact units and have high efficiency. The use of baffled anaerobic/aerobic reactors is one technological solution to provide the necessary variations in environmental conditions to foster the processes mentioned. In this paper an alternative is presented in the form of a baffled anaerobic/aerobic reactor to treat sanitary sewage in small urban and rural communities. The system performance was evaluated at several hydraulic detention times (HDT). The best values of chemical oxygen demand $\left(C O D_{\text {total }}\right)-73.7 \%$ and total suspended solids (TSS) removal - 78.8\% were obtained for HDT $_{\text {total }}$ of $8 \mathrm{~h}(4 \mathrm{~h}$ for the anaerobic phase and $4 \mathrm{~h}$ for the aerobic phase). The baffled reactor presented good operational stability, a necessary feature of low cost systems.
\end{abstract}

Key words: baffled reactor, anaerobic/aerobic process, low cost systems, rural communities

\section{INTRODUÇÃO}

Tem-se presenciado, nos avanços tecnológicos no tratamento de esgoto, a viabilização de projetos de sistemas com grande eficiência para o tratamento de águas residuárias; contudo, essas novas tecnologias estão voltadas sobretudo para o tratamento de grandes volumes de resíduos e para grandes cidades.

Nos principais centros urbanos, em particular nos das Regiões Sudeste e Sul, o solo das bacias dos rios apresenta alta 
densidade demográfica, devido à presença de indústrias. A água dos rios dessas bacias não indica características de água potabilizável, ou seja, que não demandam tratamento sofisticado. O solo é ocupado, ainda, por propriedades agrícolas que também geram produtos que poluem e contaminam os corpos de água (ou mananciais) superficiais e subterrâneos. De modo geral, pouca atenção se dá ao tratamento de efluentes líquidos gerados nas propriedades rurais, principalmente os esgotos de origem sanitária que, mesmo não apresentando concentração elevada de compostos poluidores, tem quantidade considerável lançada sem o devido tratamento nos corpos d'água mais próximos desta propriedade.

É pequena a percentagem de esgoto que pode receber qualquer tipo de tratamento, razão por que são cada vez mais sofisticados e onerosos os sistemas de tratamento das águas superficiais destinadas ao abastecimento municipal e rural. O grau de tratamento necessário depende das características dos esgotos e das possibilidades locais.

Poucos projetos de tratamento de esgoto doméstico propõem desenvolver tecnologias voltadas à população rural, ressaltando-se que os sistemas de tratamento anaeróbio de baixo custo constituem uma alternativa economicamente viável, como tanques sépticos, lagoas de estabilização, reatores anaeróbios, reúso e disposição no solo, leitos cultivados e outros.

Uma opção para solucionar o problema que as atividades zootécnicas e agrícolas causam aos recursos hídricos é o reator compartimentado anaeróbio/aeróbio. Segundo Zanella (1999) e Silva (2001) o reator compartimentado mostrou-se promissor no tratamento de águas residuárias, pela eficiência na remoção de matéria orgânica e sólidos em suspensão. pelo baixo custo de implantação e operação e pela simplicidade de operação.

Com este trabalho de pesquisa, o principal objetivo foi estudar o desempenho do reator compartimentado anaeróbio/ aeróbio para verificar a possibilidade de transferência desta tecnologia para o tratamento de águas residuárias de pequenas comunidades urbanas e rurais.

\section{MATERIAL E MÉTODOS}

Este trabalho foi realizado com o reator operando junto à estação de tratamento de esgotos (ETE) do bairro da Graminha, localizado no município de Limeira,SP, Brasil, em uma área pertencente à empresa Águas de Limeira, cedida à UNICAMP, para trabalho de pesquisa. Construído em alvenaria armada, o referido reator é formado por quatro câmaras seqüenciais: as três primeiras câmaras anaeróbias e a última câmara aeróbia, com volumes aproximados de $1,0,0,50,0,50$ e $0,50 \mathrm{~m}^{3}$, respectivamente, e com dimensões externas de $3,06 \mathrm{~m}$ de comprimento e $1,30 \mathrm{~m}$ de altura por $1,50 \mathrm{~m}$ de largura. Um decantador laminar, com volume de $0,5 \mathrm{~m}^{3}$, localizado após a câmara aeróbia completa o sistema de tratamento. O maior volume da primeira câmara tem por objetivo prolongar o tempo de detenção hidráulica nessa câmara, porque é nela que se dá a maior remoção da matéria orgânica (Clareto,1997).

O efluente líquido, que abastecia o sistema, provinha da rede coletora pública e, antes da entrada no reator compartimentado, recebe tratamento com características de nível preliminar; grade grosseira, caixa desarenadora e peneira estática. O controle da vazão de efluente para o sistema era realizado por meio de um vertedor triangular com abertura de $15^{\circ}$, localizado antes do reator (Figura 1).

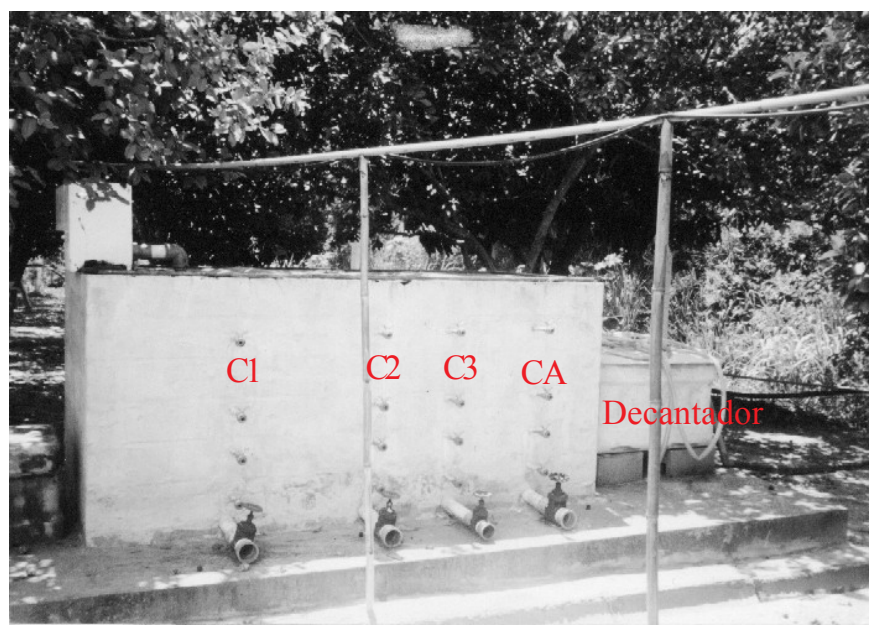

Figura 1. Reator compartimentado anaeróbio/aeróbio

Após a saída do reator colocou-se um decantador secundário laminar constituído por uma caixa de fibrocimento com $0,5 \mathrm{~m}^{3}$ de volume. A estrutura de suporte das lâminas foi confeccionada com madeira tratada com verniz naval, e as lâminas, de $250 \mathrm{~mm}$ de altura, com $2 \mathrm{~mm}$ de espessura, feitas de placas de polipropileno e inclinadas em ângulo de $60^{\circ}$, em relação à horizontal, distantes $50 \mathrm{~mm}$ entre si.

$\mathrm{O}$ fornecimento de ar para a quarta câmara foi realizado por um compressor de uso comercial com reservatório para $175 \mathrm{~L} \mathrm{e}$ pressão máxima de $120 \mathrm{lbf} \mathrm{pol}^{-2}\left(8,27 \times 10^{6} \mathrm{~N} \mathrm{~m}^{-2}\right)$, por meio de um sistema de aeração composto por difusores de ar, colocados no fundo da câmara.

Um esquema completo do sistema de tratamento utilizado no estudo pode ser observado na Figura 2.

Por processo de amostragem foi determinada a retirada de amostras nos pontos: (E) após o vertedor, antes da entrada do reator; (C3) saída da câmara 3 e localizado a 1,0 m de altura do fundo do reator; (S) após a saída do decantador laminar (Figura 2).

As amostras foram coletadas, em cada ponto, de hora em hora, durante $4 \mathrm{~h}$, uma vez por semana. Essas amostras compuseram uma amostra composta para cada ponto de amostragem. Foram estudadas as seguintes variáveis: $\mathrm{pH}$, alcalinidade total e parcial, ácidos orgânicos voláteis (AOV), demanda química de oxigênio: (DQO) total e filtrada e sólidos em suspensão totais (SST). As metodologias utilizadas para a determinação dessas variáveis estão contidas em APHA (1992), exceto para alcalinidade parcial (até $\mathrm{pH}=5,75$ ) e AOV, descritas por Ripley (1986) Dilallo (1961), respectivamente.

\section{RESULTADOS E DISCUSSÃO}

As diferentes etapas de estudo foram caracterizadas pela variação do TDH utilizado nas etapas anaeróbia e aeróbias estão descritas na Tabela 1. 


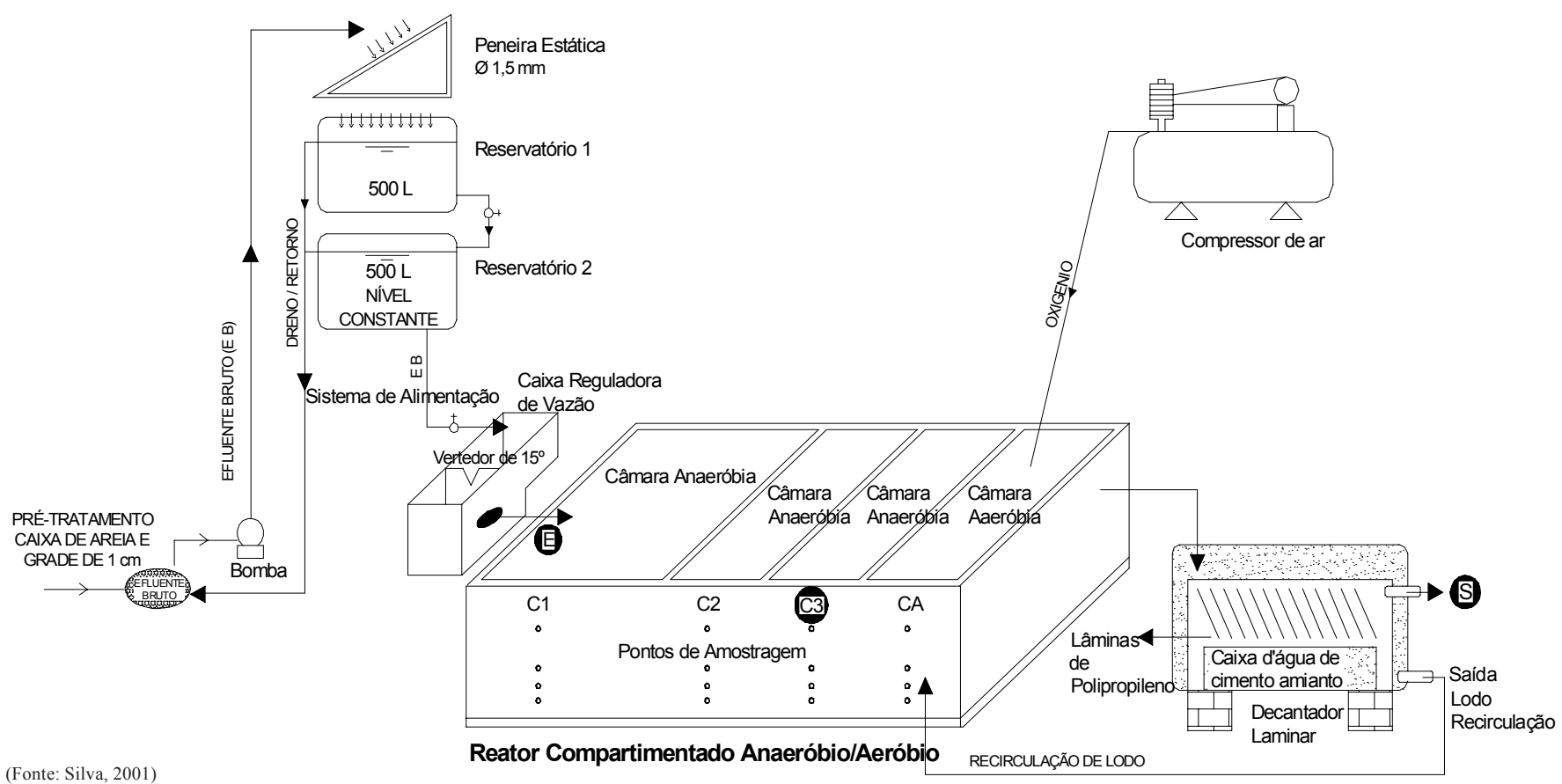

Figura 2. Esquema geral do sistema tratamento de esgotos

Tabela 1. Etapas de operação do reator durante o período de estudo

\begin{tabular}{ccccc}
\hline \multirow{2}{*}{ Etapas } & \multicolumn{4}{c}{ Tempo de operação (dia)* } \\
\cline { 2 - 5 } & CAn (h) & CAe (h) & TO (dia) & PO \\
\hline Total & - & - & 444 & $01 / 03 / 2000$ até29/05/2001 \\
$\mathrm{TDH}_{1}$ & 8 & 2 & 171 & $01 / 03 / 2000$ até $08 / 08 / 2000$ \\
$\mathrm{TDH}_{2}$ & 12 & 4 & 20 & $22 / 08 / 2000$ até $19 / 09 / 2000$ \\
$\mathrm{TDH}_{3}$ & 8 & 4 & 124 & $26 / 09 / 2000$ até $21 / 11 / 2000$ \\
$\mathrm{TDH}_{4}$ & 4 & 4 & 81 & $25 / 01 / 2001$ até $03 / 04 / 2001$ \\
$\mathrm{TDH}_{5}$ & 4 & 3 & 45 & $10 / 04 / 2001$ até $29 / 05 / 2001$ \\
\hline * CAn - Câmaras Anaeróbias; CAe - Câmaras Aeróbias; TO - Tempo de Operacãoa
\end{tabular}

* CAn - Câmaras Anaeróbias; CAe - Câmaras Aeróbias; TO - Tempo de Operação

\section{pH}

Os valores mínimos e máximos de $\mathrm{pH}$ observados durante as diferentes etapas, encontram-se na Tabela 2.

A cidade de Limeira,SP, tem, entre as suas principais atividades econômicas, a produção artesanal de bijuterias, em muitos casos realizadas por pequenas empresas caseiras. Parte dos produtos químicos utilizados para a confecção das peças é despejada na rede coletora de esgotos, sem tratamento algum (Zanella, 1999 e Camargo, 2000). Segundo esses autores, ocorreram quedas abruptas, porém esporádicas, de $\mathrm{pH}$ no esgoto bruto, atingindo valor de 1,7; contudo, durante o período de estudo não se constataram situações semelhantes, devido à correção de $\mathrm{pH}$ realizada no efluente antes da entrada no reator. Ressalta-se que nos efluentes da câmara 3, aeróbia, e da saída do reator, praticamente houve alterações de $\mathrm{pH}$, o que indica boa estabilidade e capacidade de absorção de choques pelo sistema, reforçando as pesquisas de Nachaiysit \& Stuckey (1997a, 1997b), Nour (1996), Zanella (1999), Zanella $\&$ Nour (2000), Silva et al. (2000) e Silva (2001).

Em sua maioria, os valores de $\mathrm{pH}$ apresentaram durante as cinco etapas do monitoramento, faixa de 6,7 a 7,0 , considerada ótima para processo anaeróbio, sem atingir valores prejudiciais às atividades das arqueas metanogênicas, praticamente em todo o período de operação.

\section{Alcalinidade total e parcial}

Os valores médios de alcalinidade parcial ( $\mathrm{pH}$ até 5,75) e total, na entrada e saída do sistema de tratamento, estão na Tabela 3 .

Tabela 3. Valores médios e desvio padrão* de alcalinidade total e parcial ( $\mathrm{pH}$ até 5,75$)$ na entrada e saída do sistema de tratamento, nas Etapas 1, 2, 3, 4 e $5\left(\mathrm{mg} \mathrm{CaCO}_{3} \mathrm{~L}^{-1}\right)$

\begin{tabular}{crrrc}
\hline \multirow{2}{*}{ Etapas } & \multicolumn{2}{c}{ Parcial } & \multicolumn{2}{c}{ Total } \\
\cline { 2 - 5 } & Entrada & \multicolumn{1}{c}{ Saída } & Entrada & Saída \\
\cline { 2 - 5 } & $\mathrm{M} \pm \mathrm{dp}$ & $\mathrm{M} \pm \mathrm{dp}$ & $\mathrm{M} \pm \mathrm{dp}$ & $\mathrm{M} \pm \mathrm{dp}$ \\
\hline 444 dias de operação & $100 \pm 50$ & $115 \pm 43$ & $175 \pm 73$ & $177 \pm 59$ \\
$1-\mathrm{TDH}_{1}$ & $126 \pm 45$ & $96 \pm 23$ & $212 \pm 68$ & $159 \pm 30$ \\
$2-\mathrm{TDH}_{2}$ & $77 \pm 26$ & $123 \pm 74$ & $154 \pm 31$ & $258 \pm 149$ \\
$3-\mathrm{TDH}_{3}$ & $77 \pm 25$ & $145 \pm 38$ & $145 \pm 32$ & $214 \pm 47$ \\
$4-\mathrm{TDH}_{4}$ & $83 \pm 29$ & $92 \pm 17$ & $139 \pm 44$ & $134 \pm 22$ \\
$5-\mathrm{TDH}_{5}$ & $121 \pm 86$ & $123 \pm 45$ & $205 \pm 126$ & $175 \pm 48$ \\
\hline *M $\pm \mathrm{dp}$ : valor médio, mais ou menos desvio padrão & \multicolumn{3}{c}{}
\end{tabular}

Tabela 2. Valores mínimos (mín) e máximos (máx) para pH nas etapas 1, 2, 3, 4 e 5

\begin{tabular}{ccccccc}
\hline \multirow{2}{*}{ Ponto de coleta } & 444 dias de operação & $1-\mathrm{TDH}_{1}$ & $2-\mathrm{TDH}_{2}$ & $3-\mathrm{TDH}_{3}$ & $4-\mathrm{TDH}_{4}$ & $5-\mathrm{TDH}_{5}$ \\
\cline { 2 - 7 } & mín - máx & mín - máx & mín - máx & mín - máx & mín - máx & mín - máx \\
\hline Afluente & 5,4 a 8,3 & 6,7 a 8,3 & 6,5 a 7,0 & 6,3 a 7,9 & 6,2 a 8,3 & 5,4 a 7,2 \\
Câmara 3 & 6,3 a 7,2 & 6,5 a 7,1 & 6,5 a 6,7 & 6,4 a 6,8 & 6,3 a 7,2 & 6,4 a 7,1 \\
Saída & 6,5 a 7,5 & 6,5 a 7,3 & 6,7 a 7,1 & 6,7 a 7,5 & 6,5 a 7,4 & 6,8 a 7,1 \\
\hline
\end{tabular}


Ressalta-se a importância do significado da quantificação de alcalinidade parcial e total. Alcalinidade parcial está relacionada à presença de íons bicarbonato, responsáveis pelo tamponamento do sistema no valor de $\mathrm{pH}$ desejado para as atividades biológicas; a alcalinidade total, por sua vez se correlaciona todos os compostos capazes de neutralizar ácidos incluindo-se, entre outros, os íons carbonatos, bicarbonatos e os ácidos orgânicos voláteis.

Os resultados da Tabela 3 e das Figuras 3 e 4 indicam que na Etapa 1 o reator ainda não havia atingido estabilidade adequada, a qual seria atingida somente nas Etapas 2 e 3, fato que pode ser verificado pelos valores de alcalinidade parcial de entrada e saída, respectivamente, para a Etapa 1: $126 \pm 45 \mathrm{e}$ $96 \pm 23 \mathrm{mg} \mathrm{CaCO}_{3} \mathrm{~L}^{-1}$, os quais indicam que, nesta etapa, não houve aumento na produção de íons bicarbonato. Nas demais etapas, porém, constatou-se não apenas o consumo de ácidos orgânicos voláteis mas, também, a produção de íons bicarbonato (Tabelas 3 e 4 ).

A diminuição na Etapa 3 do desvio padrão indica ter havido aumento significativo da capacidade de tamponamento do sistema, de forma tal que as variações nos valores de $\mathrm{pH}$ ocorridos no efluente bruto foram mais facilmente suportadas.

Através da Tabela 3 verifica-se, para a Etapa 5, que a diminuição ocorrida no valor médio de alcalinidade total não resultou do "consumo" de íons bicarbonato mas, sim, da menor concentração de ácidos orgânicos presentes (Tabela 4); logo, o reator apresentou manutenção significativa do sistema tampão produzido pelos íons bicarbonato, simultaneamente em consumo dos ácidos orgânicos presentes no afluente e/ou produzidos durante o metabolismo anaeróbio (Tabela 4), mesmo quando da diminuição do TDH.

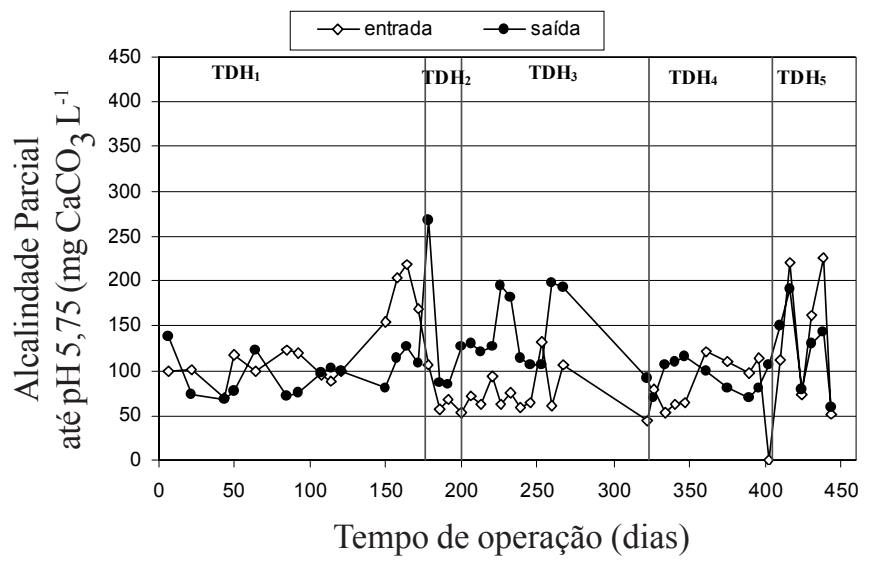

Figura 3. Alcalinidade parcial ( $\mathrm{pH}$ até 5,75) ao longo do tempo, na entrada e saída do sistema de tratamento

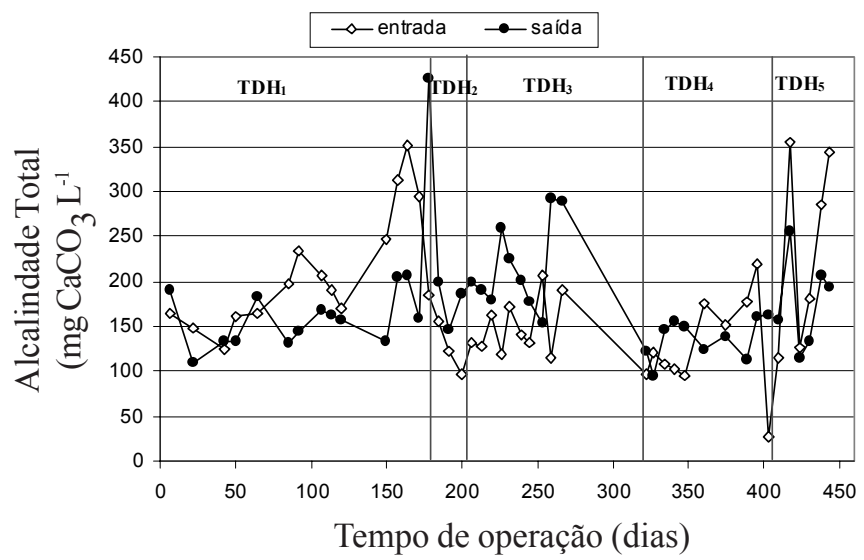

Figura 4. Alcalinidade total ao longo do tempo, na entrada e saída do sistema de tratamento

\section{Ácidos orgânicos voláteis (AOV)}

O equilíbrio na concentração dos AOV é um bom indicador do adequado funcionamento de um reator anaeróbio e o valor é de grande importância no acompanhamento da atividade das bactérias acidogênicas e arqueas metanogênicas (Tabela $4 \mathrm{e}$ Figura 5), enquanto os resultados indicam que houve atividade das arqueas metanogênicas, principalmente nas Etapas 4 e 5 , visto que a concentração de AOV na saída da câmara 3 foi significativamente menor que a do afluente ao reator.

A diminuição do TDH da Etapa 2 (12 h câmaras anaeróbias e 4 h câmara aeróbia) para a Etapa 3 ( 8 h câmaras anaeróbias e 4 h câmara aeróbia), praticamente não alterou os valores de AOV. O mesmo comportamento foi encontrado quando se diminuiu o TDH da Etapa 3 para a 4 ( 4 h câmaras anaeróbias e 4 h câmara aeróbia) ocorrendo apenas aumento no valor de entrada, característica esta apresentada pela qualidade do afluente do reator. Na Etapa 5 constatou-se aumento na concentração de AOV, na câmara 3 e na saída do sistema, o aumento decorrente de maior concentração no afluente; contudo, o valor de AOV na saída do sistema esteve próximo àqueles valores encontrados nas etapas anteriores, indicando haver estabilidade do sistema a variações nas características do afluente.

A câmara aeróbia apresentou papel significativo na remoção de AOV, como indicam os valores de concentração na saída da câmara 3 e saída do reator. Mesmo os AOV de fácil degradação biológica apresentam considerável concentração em efluentes anaeróbios, indicando a necessidade de um pós-tratamento para melhorar a qualidade desses efluentes.

Tabela 4. Valores médios e desvios padrão para ácidos orgânicos voláteis $\left(\mathrm{mg} \mathrm{HAc}^{-1}\right)$, na entrada, $3^{\mathrm{a}}$ câmara e saída do sistema de tratamento, obtidos para os pontos de coleta monitorados durante as etapas 1, 2, 3, 4 e 5

\begin{tabular}{lcccccc}
\hline \multirow{2}{*}{ Ponto de coleta } & 444 dias de operação & $\mathrm{TDH}_{1}$ & $\mathrm{TDH}_{2}$ & $\mathrm{TDH}_{3}$ & $\mathrm{TDH}_{4}$ & $\mathrm{TDH}_{5}$ \\
\cline { 2 - 7 } & $\mathrm{M} \pm \mathrm{dp}$ & $\mathrm{M} \pm \mathrm{dp}$ & $\mathrm{M} \pm \mathrm{dp}$ & $\mathrm{M} \pm \mathrm{dp}$ & $\mathrm{M} \pm \mathrm{dp}$ & $\mathrm{M} \pm \mathrm{dp}$ \\
\hline Entrada & $81 \pm 37$ & $80 \pm 14$ & $53 \pm 7$ & $57 \pm 10$ & $79 \pm 31$ & $134 \pm 56$ \\
$3^{\text {a }}$ Câmara & $68 \pm 26$ & $56 \pm 20$ & $65 \pm 13$ & $68 \pm 31$ & $68 \pm 28$ & $93 \pm 13$ \\
Saída & $43 \pm 19$ & $46 \pm 24$ & $59 \pm 25$ & $37 \pm 10$ & $34 \pm 16$ & $57 \pm 21$ \\
\hline * M \pm dp : valor médio, mais ou menos desvio padrão & & & &
\end{tabular}




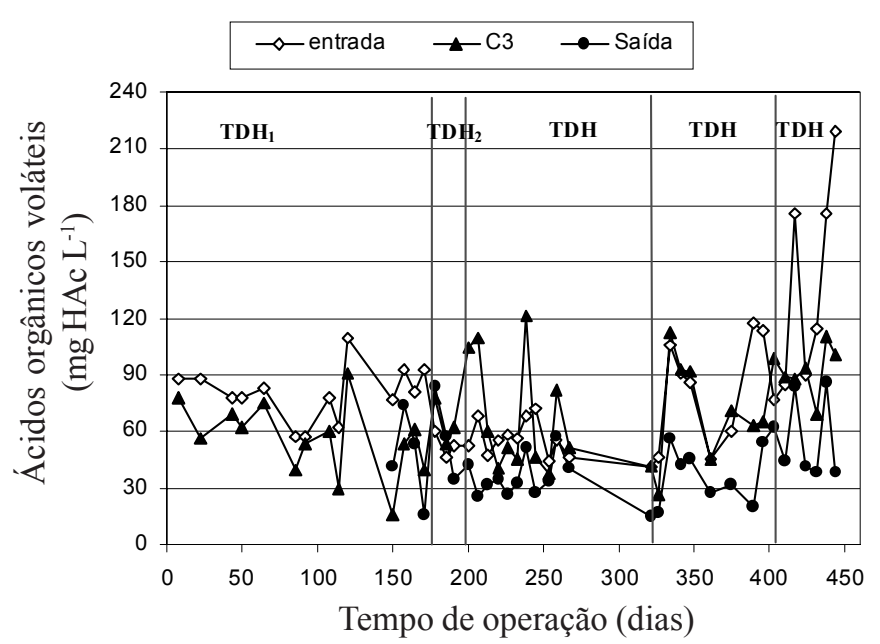

Figura 5. Ácidos orgânicos voláteis ao longo do tempo

\section{DQO $_{\text {total }}$}

Os valores de $\mathrm{DQO}_{\text {total }}$ do afluente do reator compartimentado apresentaram grande variação na Etapa 1 (Tabela 5), com a maioria dos valores compreendidos entre 450 e 1000 mg $\mathrm{O}_{2} \mathrm{~L}^{-1}$, e média e desvio padrão de $727 \pm 183 \mathrm{mg} \mathrm{O}_{2} \mathrm{~L}^{-1}$. Analisaram-se os valores de eficiência de remoção parcial definida como a eficiência de remoção de $\mathrm{DQO}_{\text {total }}$ entre a entrada do reator e a saída da $3^{a}$ câmara e remoção total definida

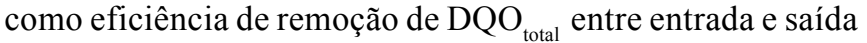
do reator, englobando câmaras anaeróbias, aeróbia e decantador secundário, cujos resultados são apresentados nas Tabela 5 e Figura 6.

Os valores médios da remoção de $\mathrm{DQO}_{\text {total }}$ estão indicados na Tabela 6 e o comportamento dessa variável, ao longo das várias etapas de operação do sistema, pode ser observado na Figura 6.

A remoção média adicional e o desvio padrão de $\mathrm{DQO}_{\text {total }}$ que a câmara aeróbia proporcionou durante as cinco fases estudadas foram de: $9 \pm 10,18 \pm 16,20 \pm 10,16 \pm 8,14 \pm 15 \%$, respectivamente; a remoção adicional média total e do desvio padrão foi de $15 \pm 12 \%$. Apesar dos resultados promissores quanto à aplicação do reator compartimentado anaeróbio/ aeróbio, a remoção de $\mathrm{DQO}_{\text {total }}$ ainda se mostrou aquém do possível em um sistema anaeróbio-aeróbio, indicando a

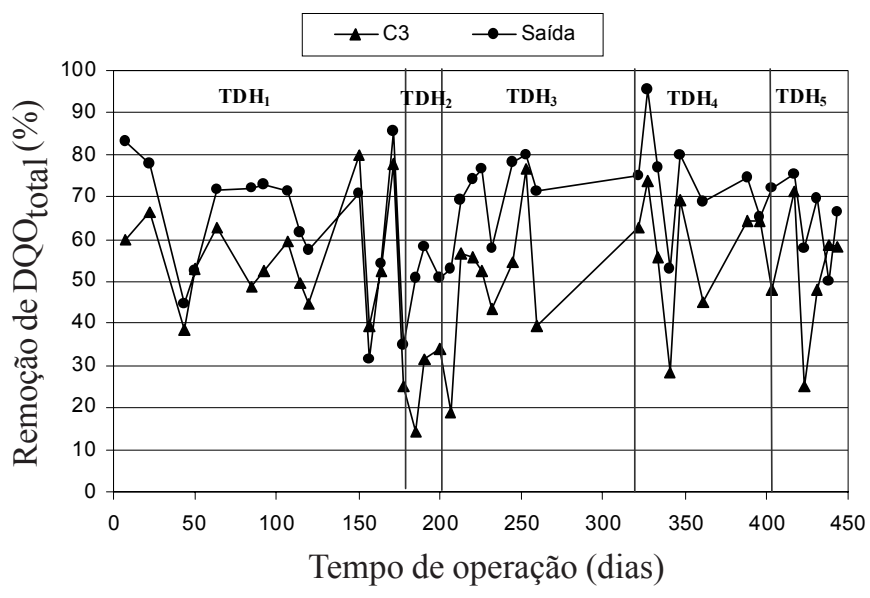

Figura 6. Porcentagem de remoção de $\mathrm{DQO}_{\text {total }}$ ao longo do tempo

necessidade de estudos adicionais com vistas à otimização do sistema de aeração, principalmente em relação às características dos aeradores a serem empregados.

$\mathrm{Na}$ Etapa 3 , mesmo com menor TDH igual a $12 \mathrm{~h}(8 \mathrm{~h}$ câmaras anaeróbias e $4 \mathrm{~h}$ câmara aeróbia), foi melhor a remoção parcial de $\mathrm{DQO}_{\text {total }}$ em relação à Etapa 2, melhor controle da vazão e, conseqüentemente, o funcionamento do sistema.

$\mathrm{O}$ aumento do TDH da câmara aeróbia de 2 para $4 \mathrm{~h}$ (Etapa 1 para a 2,3 e 4) pouco alterou a eficiência média de remoção total de $\mathrm{DQO}_{\text {total }}$ em relação aos valores médios.

Quando o TDH foi alterado de 4 para $3 \mathrm{~h}$ (Etapa 4 para 5), ocorreu pequena diminuição na eficiência média de remoção total de $\mathrm{DQO}_{\text {total }}$ quando foram analisados apenas os valores médios; esta diminuição na eficiência média é justificada pela alteração no TDH.

Povinelli (1994) realizou estudos preliminares com um dos primeiros reatores compartimentados anaeróbios construídos no Brasil, em escala piloto, para pesquisas, com volume de 11 $\mathrm{m}^{3}$, tratando esgoto sanitário. Os valores de remoção parcial de $\mathrm{DQO}_{\text {total }}$ encontrados para as Etapas 1, 4 e 5 do presente estudo, foram um pouco maiores (somente câmaras anaeróbias) que $50 \%$, valor também encontrado por este pesquisador para a partida de um reator compartimentado anaeróbio de 3 câmaras seguidas por filtro de britas.

Tabela 5. Valores médios e desvios padrão* absolutos para $\mathrm{DQO}_{\text {total }}\left(\mathrm{mg} \mathrm{O}_{2} \mathrm{~L}^{-1}\right)$

\begin{tabular}{lcccccc}
\hline \multirow{2}{*}{ Ponto de coleta } & 444 dias de operação & $1-\mathrm{TDH}_{1}$ & $2-\mathrm{TDH}_{2}$ & $3-\mathrm{TDH}_{3}$ & $4-\mathrm{TDH}_{4}$ & $5-\mathrm{TDH}_{5}$ \\
\cline { 2 - 7 } & $\mathrm{M} \pm \mathrm{dp}$ & $\mathrm{M} \pm \mathrm{dp}$ & $\mathrm{M} \pm \mathrm{dp}$ & $\mathrm{M} \pm \mathrm{dp}$ & $\mathrm{M} \pm \mathrm{dp}$ & $\mathrm{M} \pm \mathrm{dp}$ \\
\hline Entrada & $790 \pm 213$ & $764 \pm 244$ & $840 \pm 12$ & $945 \pm 255$ & $680 \pm 105$ & $742 \pm 107$ \\
$3^{\text {a Câmara }}$ & $375 \pm 139$ & $323 \pm 109$ & $641 \pm 66$ & $462 \pm 90$ & $284 \pm 105$ & $356 \pm 106$ \\
Saída & $263 \pm 115$ & $260 \pm 124$ & $437 \pm 97$ & $288 \pm 70$ & $174 \pm 82$ & $263 \pm 101$ \\
\hline " $\mathrm{M} \pm$ dp : valor médio, mais ou menos desvio padrão & & & & &
\end{tabular}

Tabela 6. Valores médios e desvios padrão* da porcentagem de remoção de $\mathrm{DQO}_{\text {total }}$ na $3^{\mathrm{a}}$ câmara e saída do sistema de tratamento obtidos durante as etapas $1,2,3,4$ e 5

\begin{tabular}{lcccccc}
\hline \multirow{2}{*}{ Ponto de coleta } & 444 dias de operação & $1-\mathrm{TDH}_{1}$ & $2-\mathrm{TDH}_{2}$ & $3-\mathrm{TDH}_{3}$ & $4-\mathrm{TDH}_{4}$ & $5-\mathrm{TDH}_{5}$ \\
\cline { 2 - 7 } & $\mathrm{M} \pm \mathrm{dp}$ & $\mathrm{M} \pm \mathrm{dp}$ & $\mathrm{M} \pm \mathrm{dp}$ & $\mathrm{M} \pm \mathrm{dp}$ & $\mathrm{M} \pm \mathrm{dp}$ & $\mathrm{M} \pm \mathrm{dp}$ \\
\hline $3^{\text {a Câmara }}$ & $52 \pm 16$ & $56 \pm 13$ & $24 \pm 9$ & $48 \pm 16$ & $58 \pm 15$ & $52 \pm 7$ \\
Saída & $66 \pm 14$ & $65 \pm 15$ & $48 \pm 12$ & $68 \pm 11$ & $74 \pm 12$ & $65 \pm 10$ \\
\hline
\end{tabular}

* $\mathrm{M} \pm \mathrm{dp}$ : valor médio, mais ou menos desvio padrão 
Nour (1996), estudou um reator compartimentado anaeróbio de $11 \mathrm{~m}^{3}$ e quatro compartimentos, tratando esgoto sanitário, em três etapas ( $1^{\text {a }}$ Etapa -12 h de TDH e duração de 296 dias; $2^{\mathrm{a}}$ Etapa $-8 \mathrm{~h}$ de TDH e duração de 266 dias; $3^{\mathrm{a}}$ Etapa $-12 \mathrm{~h}$ de TDH e duração de 91 dias). Seus resultados apresentaram remoção total e desvios padrão de $\mathrm{DQO}_{\text {total }}$ para as 3 etapas estudadas, $\mathrm{TDH}_{1}=12 \mathrm{~h}$ (Etapa $\left.1 \mathrm{~B}\right), \mathrm{TDH}_{2}=8 \mathrm{~h}($ Etapa 2$) \mathrm{e}$ $\mathrm{TDH}_{3}=12 \mathrm{~h}$ (Etapa 3 ) e os seguintes valores: $52,5 \pm 8,6 \%, 42,1$ $\pm 13,9 \%$ e $56,7 \pm 9,5 \%$, respectivamente. Comparando-se apenas os valores médios de remoção de $\mathrm{DQO}_{\text {total }}$, os resultados obtidos por Nour (1996) em reator no qual havia somente câmaras anaeróbias, foram menores que os encontrados no presente estudo, para as cinco etapas.

Zanella (1999) com o mesmo reator utilizado nesta pesquisa, estudou a partida de um reator compartimentado anaeróbio/ aeróbio. O sistema foi operado durante 152 dias, com três TDH diferentes, 30, 17,5 e $10 \mathrm{~h}$, consecutivamente. Seu trabalho apresentou valor de remoção média total e desvio padrão de $\mathrm{DQO}_{\text {total }}$ de $72,3 \pm 9,9 \%$, para o $\mathrm{TDH}_{\text {total }}=10 \mathrm{~h}$. Verifica-se semelhança dos valores médios obtidos por Zanella (1999) e os valores médios da Etapa 4 desta pesquisa, realizado com TDH melhor.

\section{DQO filtrada $_{\text {affuent }}$}

$\mathrm{O}$ afluente (efluente bruto) apresentou alta amplitude de variação para os valores de $\mathrm{DQO}_{\text {filtrada }}$ (Tabela 7), comportamento idêntico ao apresentado pela $\mathrm{DQO}_{\text {total }}$; tais valores se situaram na faixa de 215 a $603 \mathrm{mg} \mathrm{O}_{2} \mathrm{~L}^{-1}$ durante todo o período experimental. Considerando-se cada etapa de operação, foram encontradas as faixas: para a Etapa 1 de 274 a $303 \mathrm{mg} \mathrm{O}_{2} \mathrm{~L}^{-1}$, Etapa 2 de 366 a $533 \mathrm{mg} \mathrm{O}_{2} \mathrm{~L}^{-1}$, para a Fase $3 \mathrm{de}$ 227 a $603 \mathrm{mg} \mathrm{O}_{2} \mathrm{~L}^{-1}$, para Etapa 4 de 215 a $350 \mathrm{mg} \mathrm{O}_{2} \mathrm{~L}^{-1}$ e para a Etapa 5 de 254 a $481 \mathrm{mg} \mathrm{O}_{2} \mathrm{~L}^{-1}$.

$\mathrm{O}$ afluente (efluente bruto) apresentou alta amplitude de variação para os valores de $\mathrm{DQO}_{\text {filtrada }}$, comportamento semelhante ao apresentado pela $\mathrm{DQO} \mathrm{total}$. Os valores médios de remoção de $\mathrm{DQO}_{\text {filtrada }}$ estão representados na Tabela 8 e o comportamento da remoção de $\mathrm{DQO}_{\text {filtrada }}$ pode ser observado na Figura 7.

Para a Etapa 1, a DQO filtrada apresentou tendência similar a $\mathrm{DQO}_{\text {total }}$, porém com maior remoção da $\mathrm{DQO}_{\text {filtrada }}$ em relação a
$\mathrm{DQO}_{\text {total }}$, como pode ser observado nas Tabelas 6 e 8 e nas Figuras 6 e 7 .

Mesmo com a diminuição do TDH das câmaras anaeróbias de 12 para 8 h (Etapa 2 para a 3) não se verificou queda na efíciência média de remoção parcial de $\mathrm{DQO}_{\text {filtrada }}$ mas, sim, aumento nos valores médios de remoção parcial. O mesmo ocorreu quando se diminuiu o TDH da Etapa anaeróbia, de 8 para 4 h (Etapa 3 para a 4 ) com aumento de 45 para $54 \%$ na eficiência de remoção, cujo resultado indica que o reator compartimentado anaeróbio/aeróbio apresenta boa flexibilidade e rápida absorção de choques ocasionados pela variação do

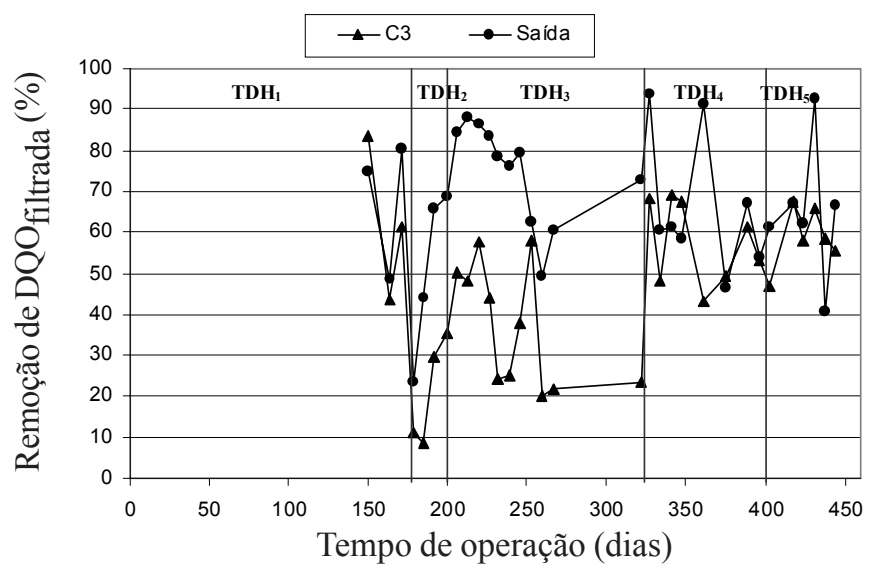

Figura 7. Porcentagem de remoção de $\mathrm{DQO}_{\text {filtrada }}$ ao longo do tempo

TDH. A Etapa 5 não indicou diferença significativa no valor de remoção parcial de $\mathrm{DQO}_{\text {filtrada }}$.

Lopes (1999), operando um reator compartimentado aeróbio no qual era recebido como afluente o efluente de um reator compartimentado anaeróbio, com $\mathrm{TDH}_{\text {total }}=8 \mathrm{~h}$ obteve $81 \pm 7 \%$ de remoção média de $\mathrm{DQO}_{\text {filtrada }}$, $\mathrm{TDH}_{\text {total }}=6 \mathrm{~h}$, obteve $79 \pm$ $12 \%$ - valores esses superiores aos encontrados no presente estudo, ressaltando-se, porém, que a autora operou um reator de bancada no qual se conseguia controlar melhor as variáveis do sistema o que, sem dúvida, possibilitou o alto valor da eficiência de remoção, comparado aos valores encontrados nesta pesquisa em que se operou reator compartimentado.

Tabela 7. Valores médios e desvios padrão da porcentagem de remoção para $\mathrm{DQO}_{\text {filtrada }}\left(\mathrm{mg} \mathrm{O}_{2} \mathrm{~L}^{-1}\right)$

\begin{tabular}{lccccrc}
\hline \multirow{2}{*}{ Ponto de coleta } & 444 dias de operação & $1-\mathrm{TDH}_{1}$ & $2-\mathrm{TDH}_{2}$ & $3-\mathrm{TDH}_{3}$ & $4-\mathrm{TDH}_{4}$ & $5-\mathrm{TDH}_{5}$ \\
\cline { 2 - 7 } & $\mathrm{M} \pm \mathrm{dp}$ & $\mathrm{M} \pm \mathrm{dp}$ & $\mathrm{M} \pm \mathrm{dp}$ & $\mathrm{M} \pm \mathrm{dp}$ & $\mathrm{M} \pm \mathrm{dp}$ & $\mathrm{M} \pm \mathrm{dp}$ \\
\hline Entrada & $336 \pm 90$ & $292 \pm 10$ & $385 \pm 108$ & $369 \pm 111$ & $283 \pm 46$ & $342 \pm 76$ \\
$3^{\text {a Câmara }}$ & $201 \pm 118$ & $189 \pm 152$ & $285 \pm 122$ & $264 \pm 135$ & $130 \pm 53$ & $142 \pm 40$ \\
Saída & $118 \pm 75$ & $175 \pm 114$ & $181 \pm 94$ & $99 \pm 75$ & $92 \pm 44$ & $123 \pm 62$ \\
F $\mathrm{M} \pm$ dp : valor médio, mais ou menos desvio padrão & & & &
\end{tabular}

Tabela 8. Valores médios da porcentagem de remoção de $\mathrm{DQO}_{\text {filtrada }}$ na $3^{\mathrm{a}}$ câmara e saída do sistema de tratamento obtidos durantes as Etapas 1, 2,3, 4 e 5

\begin{tabular}{lcccccc}
\hline \multirow{2}{*}{ Ponto de coleta } & 444 dias de operação & $1-\mathrm{TDH}_{1}$ & $2-\mathrm{TDH}_{2}$ & $3-\mathrm{TDH}_{3}$ & $4-\mathrm{TDH}_{4}$ & $5-\mathrm{TDH}_{5}$ \\
\cline { 2 - 7 } & $\mathrm{M} \pm \mathrm{dp}$ & $\mathrm{M} \pm \mathrm{dp}$ & $\mathrm{M} \pm \mathrm{dp}$ & $\mathrm{M} \pm \mathrm{dp}$ & $\mathrm{M} \pm \mathrm{dp}$ & $\mathrm{M} \pm \mathrm{dp}$ \\
\hline $3^{\mathrm{a}}$ Câmara & $47 \pm 19$ & $53 \pm 13$ & $16 \pm 11$ & $45 \pm 8$ & $54 \pm 15$ & $59 \pm 7$ \\
Saída & $67 \pm 17$ & $65 \pm 22$ & $44 \pm 21$ & $80 \pm 10$ & $67 \pm 16$ & $65 \pm 19$ \\
\hline
\end{tabular}

* $\mathrm{M} \pm \mathrm{dp}$ : valor médio, mais ou menos desvio padrão 


\section{Sólidos em suspensão totais (SST)}

Na Tabela 9 apresenta os valores médios de sólidos em suspensão total.

Os valores de remoção de sólidos em suspensão total obtidos estão apresentadas na Tabela 10 e o comportamento da remoção de SST pode ser observado na Figura 8.

A diminuição do TDH das câmaras anaeróbias, de 12 para 8 h (Etapa 2 para a 3), não diminuiu mas aumentou a eficiência média de remoção parcial de SST. O mesmo foi verificado na mudança do TDH das câmaras anaeróbias da Etapa 3 para a 4, na qual se diminuiu o TDH em $50 \%$. Na Etapa 5 foi mantido o tempo de $4 \mathrm{~h}$ para as câmaras anaeróbias.

Os resultados apresentados na Tabela 10 indicam que o aumento do TDH da câmara aeróbia, de 2 para 4 h (Etapa 1 para as 2,3 e 4) pouco alterou a eficiência média de remoção total de SST.

Com a alteração do TDH de 4 para 3 h (Etapa 4 para a 5) ocorreu pequeno decréscimo na eficiência média de remoção de SST, ocasionando, possivelmente, pela quebra da bomba que recalcava o esgoto para as caixas de alimentação.

Lopes (1999), na segunda etapa de seu estudo, em reator compartimentado aeróbio alimentado com efluente de um reator compartimentado anaeróbio e utilizando um TDH de 8 e de 6 h,

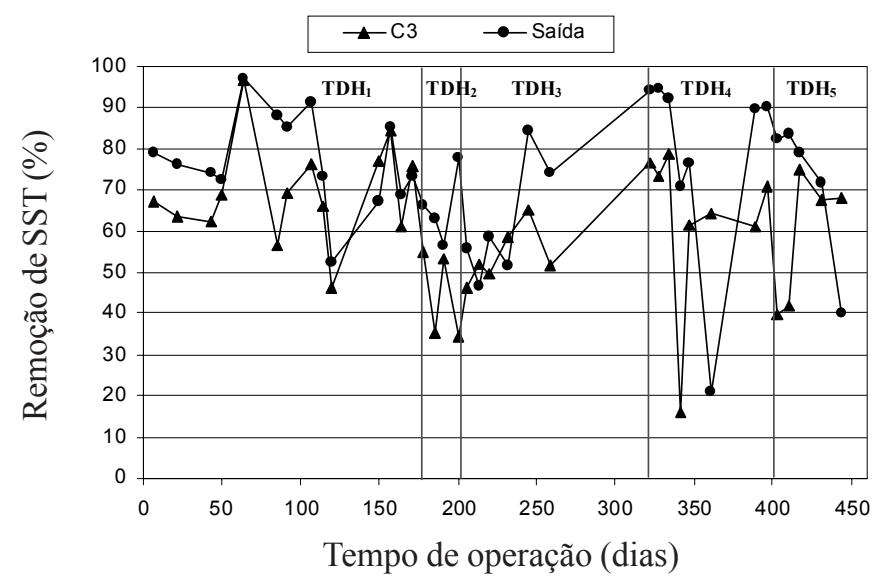

Figura 8. Eficiência de remoção de SST ao longo do tempo obteve remoção média e desvio padrão de SST iguais a 94,0 \pm 5,0 e $82 \pm 20 \%$ respectivamente, valores esses maiores que os valores encontrados neste estudo.

Ressalta-se que durante a diminuição do TDH não houve alteração no desempenho do reator em relação à remoção de SST; aliás, este procedimento proporcionou adaptação de biomassa diante de novas condições de operação.

\section{Consumo de Energia}

A configuração proposta para o reator compartimentado anaeróbio/aeróbio, mostra a necessidade de tratamento de águas residuárias geradas em edificações e/ou instalações localizadas na zona rural. A necessidade de aeração na quarta câmara do reator e, conseqüentemente, de energia elétrica para o funcionamento do compressor de ar, não inviabiliza seu uso como sistema de baixo custo para o tratamento de águas residuárias. Ressalta-se que a moderna atividade rural, que viabiliza a manutenção do homem no campo, necessita e utiliza a energia elétrica em todas as atividades correlatas. Os problemas ambientais causados por água residuárias provenientes de diferentes atividades em propriedades rurais, possivelmente poderão ser minimizados com o uso do reator compartimentado anaeróbio/aeróbio, o qual não apresenta dificuldades de construção e operação.

Com o objetivo de calcular o consumo médio diário de energia por habitante do sistema de tratamento, fez-se uma comparação com o consumo de energia de um chuveiro elétrico de $3,5 \mathrm{~kW}$, usado por uma família de 4 pessoas, cada qual o utilizando $10(\mathrm{dez})$ min por dia (Tabela 11).

Os resultados contidos na Tabela 11 indicam menor consumo de energia do compressor de ar comparado a um chuveiro elétrico comumente utilizado em residência. Este fato vem indicar a viabilidade da utilização de um sistema de aeração composto por um compressor de ar, facilmente encontrado no comércio.

O sistema estudado poderia ser usado por 40 pessoas consumindo, em média $150 \mathrm{~L} \mathrm{hab}^{-1} \mathrm{dia}^{-1}, \mathrm{com} \mathrm{um} \mathrm{TDH}_{\text {total }}=10 \mathrm{~h}$.

Tabela 9. Valores médios e desvios padrão absolutos para SST (mg L-1)

\begin{tabular}{lcccccc}
\hline \multirow{2}{*}{ Ponto de coleta } & 444 dias de operação & $1-\mathrm{TDH}_{1}$ & $2-\mathrm{TDH}_{2}$ & $3-\mathrm{TDH}_{3}$ & $4-\mathrm{TDH}_{4}$ & $5-\mathrm{TDH}_{5}$ \\
\cline { 2 - 7 } & $\mathrm{M} \pm \mathrm{dp}$ & $\mathrm{M} \pm \mathrm{dp}$ & $\mathrm{M} \pm \mathrm{dp}$ & $\mathrm{M} \pm \mathrm{dp}$ & $\mathrm{M} \pm \mathrm{dp}$ & $\mathrm{M} \pm \mathrm{dp}$ \\
\hline Entrada & $279 \pm 61$ & $282 \pm 54$ & $290 \pm 68$ & $305 \pm 58$ & $235 \pm 46$ & $351 \pm 37$ \\
$3^{\text {a Câmara }}$ & $108 \pm 49$ & $79 \pm 29$ & $151 \pm 44$ & $145 \pm 19$ & $84 \pm 34$ & $149 \pm 70$ \\
Saída & $73 \pm 39$ & $58 \pm 28$ & $108 \pm 2$ & $117 \pm 33$ & $40 \pm 27$ & $83 \pm 33$ \\
\hline * M + dp . valor médio, mais ou menos desvio padrão & & &
\end{tabular}

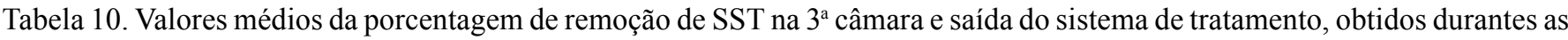
etapas $1,2,3,4$ e 5

\begin{tabular}{lcccccc}
\hline \multirow{2}{*}{ Ponto de coleta } & 444 dias de operação & $1-\mathrm{TDH}_{1}$ & $2-\mathrm{TDH}_{2}$ & $3-\mathrm{TDH}_{3}$ & $4-\mathrm{TDH}_{4}$ & $5-\mathrm{TDH}_{5}$ \\
\cline { 2 - 7 } & $\mathrm{M} \pm \mathrm{dp}$ & $\mathrm{M} \pm \mathrm{dp}$ & $\mathrm{M} \pm \mathrm{dp}$ & $\mathrm{M} \pm \mathrm{dp}$ & $\mathrm{M} \pm \mathrm{dp}$ & $\mathrm{M} \pm \mathrm{dp}$ \\
\hline $3^{\text {a }}$ Câmara & $61 \pm 16$ & $69 \pm 12$ & $48 \pm 11$ & $54 \pm 10$ & $63 \pm 20$ & $58 \pm 16$ \\
Saída & $73 \pm 17$ & $77 \pm 11$ & $62 \pm 5$ & $65 \pm 16$ & $79 \pm 25$ & $71 \pm 18$ \\
\hline * $\mathrm{M} \pm$ dp : valor médio, mais ou menos desvio padrão & & & &
\end{tabular}


Tabela 11. Valores médios* do consumo por hab dia ${ }^{-1}$, do sistema de tratamento, e de um chuveiro elétrico utilizado por família de 4 pessoas

\begin{tabular}{lcccc}
\hline Equipamento & $\begin{array}{c}\text { Potencia } \\
(\mathrm{kW})\end{array}$ & $\begin{array}{c}\mathrm{TU} \\
\left(\mathrm{h} \mathrm{dia}^{-1}\right)\end{array}$ & $\begin{array}{c}\text { PA } \\
(\text { habitante })\end{array}$ & $\begin{array}{c}\text { Consumo } \\
(\mathrm{kWh} / \mathrm{hab} \text {. dia })\end{array}$ \\
\hline Compressor de ar & 1,5 & 12 & 40 & 0,45 \\
Chuveiro elétrico & 3,5 & 0,67 & 4 & 0,59 \\
\hline * TU - Tempo de utilização; PA - População atendida & &
\end{tabular}

\section{CONCLUSÕES}

1. Independentemente do valor de entrada, o valor de $\mathrm{pH}$ da saída do reator também se manteve próximo de 7,0 durante as cinco etapas, indicando boa estabilidade do reator compartimentado anaeróbio/aeróbio.

2. A configuração do reator mostra boa capacidade para absorver choques hidráulicos e orgânicos, sem provocar qualquer instabilidade no sistema. Este fato pode ser observado no comportamento do reator quando da diminuição dos valores TDH, com conseqüente aumento na carga orgânica aplicada.

3. O reator apresentou boa flexibilidade quanto às mudanças de TDH, no que se refere às eficiências de remoção de $\mathrm{DQO}_{\text {total }}$, $\mathrm{DQO}_{\text {filtrada }}, \mathrm{DBO}_{5}$ e SST.

4. Configuração simples, construção simples e facilidade operacional, são vantagens para uso em pequenas comunidades urbanas e rurais.

5. Apesar dos resultados promissores quanto à aplicação do reator compartimentado anaeróbio/aeróbio, a remoção de $\mathrm{DQO}_{\text {total }}$ se mostrou aquém de possível em um sistema anaeróbio-aeróbio, apontando a necessidade de estudos adicionais com vistas à otimização dos sistemas.

6. O consumo de energia diário do compressor de ar por habitante, utilizado no sistema de tratamento, é menor que o consumo diário de um chuveiro por habitante.

7. O sistema estudado nesta pesquisa poderia ser usado por 40 pessoas consumindo em média $150 \mathrm{~L} \mathrm{hab}^{-1} \mathrm{dia}^{-1}$, com um $\mathrm{TDH}_{\text {total }}=10 \mathrm{~h}$.

\section{LITERATURA CITADA}

APHA; AWWA.; WPCF, Standard Methods for the Examination of Water and Wastewater. 18. ed. Washington D. C., 1992

Camargo, S.A.R. Filtro anaeróbio com enchimento de bambu para tratamento de esgotos sanitários: Avaliação da Partida e Operação, Campinas: UNICAMP, 2000, 181p. Dissertação Mestrado
Clareto, C.R. Tratamento biológico de líquidos percolados gerados em aterros sanitários utilizando reator anaeróbio compartimentado. São Carlos: Escola de Engenharia de São Carlos: USP, 1997, 300p. Dissertação Mestrado

DiLallo, R.; Albertson, O.E., Volatile acids by direct titration, Journal WPCF, Alexandria, US, v.33, n.4, p.356-365, 1961.

Lopes, D.D. Tratamento de esgoto sanitário empregando-se reatores compartimentados anaeróbio/aeróbio em série. São Carlos: Escola de Engenharia de São Carlos: USP, 1999, 178p. Tese Doutorado

Nachaiyasit, S.; Stuckey, D.C. The effect of shock load on the performance of anaerobic baffled reactor (ABR). 1. Steps changes in feed concentration at constant retention time. Water Research, New York, v.31, n.11, p.2737-2746, 1997a.

Nachaiyasit, S.; Stuckey, D.C. The effect of shock load on the performance of anaerobic Baffled reactor (ABR). 2. Step and transient hydraulic shocks at constant feed strength. Water Research, New York, v.31, n.11, p.2747-2754, 1997 b.

Nour, E.A.A. Tratamento de esgoto sanitário empregando-se reator compartimentado. São Carlos: USP, 1996, 266p. Tese Doutorado

Povinelli, S.C.S. Estudo da hidrodinâmica e partida de reator anaeróbio com chicanas tratando esgoto sanitário. São Carlos: Escola de Engenharia de São Carlos, USP, 1994, 128p. Dissertação Mestrado

Ripley, L.E.; Boyle, W.C.; Converse, J.C. Improved alkalimetric monitoring for anaerobic digestion of high-strength wastes. Journal WPCF, Alexandria, v.38, n.35, p.406-411, 1986.

Silva, G.H.R. Reator compartimentado anaeróbio/aeróbio tratando esgoto sanitário: Desempenho e Operação. Campinas: UNICAMP, 166p. 2001. Dissertação Mestrado

Silva, G.H.R.; Zanella, L.; Nour, E.A.A. Comportamento de um reator compartimentado híbrido submetido a um período de operação precária: Estabilidade do Sistema, Congresso Interamericano de Ingenieria Sanitária y Ambiental, 17, Porto Alegre, Anais Eletrônicos, 2000.

Zanella, L. Partida de um reator compartimentado híbrido anaeróbio/aeróbio tratando esgoto sanitário. Campinas: UNICAMP, 1999. 118p. Dissertação Mestrado

Zanella, L.; Nour, E.A.A. Partida de um reator compartimentado híbrido anaeróbio/aeróbio tratando esgoto sanitário In: Latin American Workshop and Seminar on Anaerobic Digestion 6, 2000, Recife, Anais, v. 2 , n.1, p.195-198. 